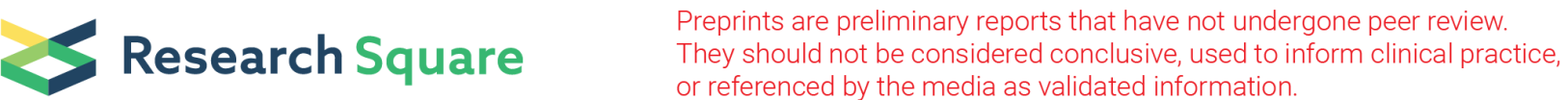

\section{RNA-seq analysis of ischemia stroke and normal brain in a tree shrew model with or without type 2 diabetes mellitus}

\section{Ling Zhao}

Department of Endocrinology, the second affiliated hospital of Kunming medical university

\section{Shiying Huang}

Department of Endocrinology, the first affiliated hospital of kunming medical university

\section{Qiwei Liao}

Department of Cardiology, The Yan-an affiliated hospital of Kunming medical university

\section{Xia Li}

Department of Pathophysiology, Kunming medical university

\section{Shufen Tan}

Department of Gynecologic Oncology, the third affiliated hospital of Kunming medical university

\section{Shuqing Li}

Department of Pathophysiology, Kunming medical university

Tingyu Ke ( $\sim 34078910 @ q q . c o m$ )

The Second Affiliated Hospital of Kunming Medical University https://orcid.org/0000-0001-8848-5441

\section{Research Article}

Keywords: RNA-seq analysis, type 2 diabetes mellitus, ischemia stroke, tree shrew model

Posted Date: June 17th, 2021

DOI: https://doi.org/10.21203/rs.3.rs-170576/v1

License: (a) (i) This work is licensed under a Creative Commons Attribution 4.0 International License. Read Full License

Version of Record: A version of this preprint was published at Metabolic Brain Disease on August 21st, 2021. See the published version at https://doi.org/10.1007/s11011-021-00813-5. 


\section{Abstract}

Background: Nowadays, similar strategies have been used for the treatment and prevention of acute stroke in both diabetes mellitus (DM) and non-DM populations. These strategies were analyzed to provide an experimental basis for the clinical prevention and treatment of stroke in patients both with and without DM.

Methods: Tree shrews were randomly divided into control, DM, ischemic stroke (IS), and DMIS groups with 18 animals in each group. Serum biochemical indicators were used to assess metabolic status. Neural tissue damage was determined using triphenyl tetrazolium chloride staining, $\mathrm{H}$-E staining, and electron microscopy. Differential gene expression of neural tissue between the DM and control groups and the IS and DMIS groups was measured using RNA-seq analysis.

Results: The serum glucose levels of the DM and DMIS groups were significantly higher than other groups. In the DMIS group, the infarct size was significantly larger than in the IS group (19.56 $\pm 1.25 \%$ ), with a more obvious abnormal ultrastructure of neural cells. RNA-seq analysis showed that the expression of IL-8, C-C motif chemokine 2 (CCL2), and alpha-1-antichymotrypsin was significantly higher in the DM group than in the control group. The CCL7, ATP-binding cassette sub-family A member 12, and adhesion G protein-coupled receptor E2 levels were significantly higher in the DMIS group than in the IS group.

Conclusions: For the prevention and treatment of stroke in patients with DM, reducing the inflammatory state of the nervous system may reduce the incidence of stroke and improve the prognosis of neurological function after IS.

\section{Introduction}

Stroke is the leading cause of adult disability and mortality worldwide (Collaborators GBDS, 2019). Diabetes mellitus (DM) is an important risk factor for stroke, and patients with DM are 2-6 times more likely to experience a stroke (Jiang et al, 2018; Ma et al, 2018). In addition, approximately 20-30\% of in-hospital patients with stroke also had DM, and 90\% had type 2 DM (Hill 2014, Beckman et al, 2013) Stroke and DM have similar risk factors, including smoking, a sedentary lifestyle, obesity, a high-fat diet, and alcoholism (Hong et al, 2019). DM is also an important factor in determining post-stroke neural functional recovery. As a major concomitant disease, DM can aggravate brain tissue damage after ischemia, leading to worse neurological outcomes (Bhalla et al, 2013). DM is also correlated with larger infarct size and worse neurobehavioral outcomes (Ma et al, 2018; Hill 2014; Pintana et al, 2019). Patients with acute stroke with a blood glucose level greater than $8 \mathrm{mM}$ have a nearly two-fold mortality rate and a greater likelihood of complications than patients with a blood glucose level lower than $8 \mathrm{mM}$ (Ma et al, 2018; Romero et al, 2008). DM does not affect the severity of stroke at the initial stage, but is an independent influencing factor for neurological function in the long term (Liu et al, 2020).

DM may also affect the efficacy of drugs used in stroke therapy. For patients with $\mathrm{DM}$, acute thrombolytic therapy was more likely to cause hemorrhagic transformations than for those without DM (Wen et al, 2020). Furthermore, thrombolytic therapy might not be the best treatment for stroke patients with hyperglycemia (Bruno et al, 2010). Therefore, the presence of DM affects the therapeutic strategies used to treat stroke. 
Although current guidelines recommend strategies for treating hyperglycemia during a stroke, these are primarily based on expert opinion (Kruyt et al, 2010). Therefore, the development of targeted effective treatment strategies for stroke patients with diabetes is the focus of the current study. High blood glucose affects the integrity of the white matter of the brain, the production of oligodendrocytes, and the reduction of neural plasticity during post-stroke recovery (Hill 2014; Pintana et al, 2019). Therefore, high blood glucose levels may cause pro-inflammatory morphology in microglial cells and macrophages. However, the molecular mechanism for this phenomenon is not yet fully understood.

At present, similar strategies have been used for the treatment and prevention of acute stroke in both DM and non-DM populations. However, it is necessary to identify different treatments for stroke patients both with and without DM, as well as appropriate prevention techniques. In this study, tree shrews were used to establish DM and stroke models, and RNA-seq and bioinformatics analyses were performed to detect the gene expression characteristics of brain tissue in each group. Comparisons between the DM and control models and between the DM stroke and control stroke models were made to provide an experimental basis for the identification of clinical prevention techniques and treatments of stroke in patients with DM.

\section{Materials And Methods}

\section{Animal models and groups}

Adult male tree shrews weighing $130 \pm 30 \mathrm{~g}$ were given free access to food and water. The cultivation environment was natural light for $12 \mathrm{~h}$ at $25 \pm 3^{\circ} \mathrm{C}$. Adaptive feeding occurred one week before experimentation began. The tree shrews were randomly divided into four groups, namely, the control, DM, ischemia stroke (IS), and DMIS, with 18 animals per group (Figure 1).

The DM model was established using the method described subsequently. High-fat feed (basic feed $+1 \%$ cholesterol $+0.1 \%$ sodium cholate $+10 \%$ lard $+5 \%$ egg yolk powder $+5 \%$ whole milk powder, Dashuo Chengdu, China) was fed to the tree shrews for eight weeks. After the injection of $2 \%$ streptozotocin (STZ, Sigma-Aldrich, USA) into the femoral vein at $100 \mathrm{mg} / \mathrm{kg}$ dose, blood glucose changes in the tree shrews were dynamically monitored on days 3,7 , and 14 . When the blood glucose exceeded $16.7 \mathrm{mmol} / \mathrm{L}$, the DM model was considered successful and was able to maintain hyperglycemia for four weeks. This model induced insulin resistance and established risk factors, such as high fat and high cholesterol levels, which are close to clinical DM characteristics. Animals in the control and IS groups were injected with sodium citrate.

The thrombotic cerebral IS model was induced using photochemical treatment. After anesthetizing, tree shrews received a $1-\mathrm{cm}$ incision at the midpoint of the connection between the right tragus and the outer canthus of the right eye. The temporalis muscle was separated to expose the skull. A sterile aluminum sheet with a $0.5-\mathrm{cm}$ diameter circular window hole was embedded in the incision. Then, $1.33 \mathrm{~mL} / \mathrm{kg}$ Bengal red saline solution was injected into the femoral vein for $10 \mathrm{~min}$. A SQ-III cerebral thrombosis device (Chinese patent number: ZL201420068737.2) was used to irradiate the surface of the skull through the sheet hole with a light intensity of $1.0 \mathrm{~W} / \mathrm{cm}^{2}$ (Figure 2). This light beam with a $560 \mathrm{~nm}$ wavelength $(\lambda)$ penetrated the skull to produce a photochemical reaction with Bengal red and oxygen in the cerebral blood vessels. This reaction damaged the vascular endothelium and induced thrombosis (Zhao et al, 2019). The regulation of 
ischemic postconditioning on metabolic disorders of type $2 \mathrm{DM}$ combined with cerebral ischemia in tree shrews was investigated. The control and DM groups were subjected to sham operations without

photochemical reaction. The experimental process was approved, supervised, and inspected by the Kunming Medical University Laboratory Animal Ethics Committee.

\section{Serum biochemical indicators detection}

After anesthetizing the animals in each group, blood was collected from the main abdominal vein and loaded into a coagulation tube. After centrifugation at $3000 \mathrm{rpm}$ for $10 \mathrm{~min}$, the sample was stored at $-80^{\circ} \mathrm{C}$ until further use. The biochemical indicators that were measured included glucose, lipids, C-reactive protein, total serum protein, bilirubin, albumin, globulin, alanine aminotransferase, aspartate aminotransferase, urea, creatinine, uric acid, triglycerides, total cholesterol, and high- and low-density lipoprotein cholesterol levels.

\section{Triphenyl tetrazolium chloride (TTC) staining detection}

Five models in each group were randomly selected. After $24 \mathrm{~h}$ of IS modeling, 10\% formaldehyde was infused after anesthetizing. The brain was sectioned coronally, subjected to gradient dehydration, embedded in paraffin, and stained with $\mathrm{H}-\mathrm{E}$ after sample slicing. In addition, five models were randomly selected in each group. After $24 \mathrm{~h}$ of IS modeling, the entire brain was retrieved after anesthesia and craniotomy and placed in $2 \%$ TTC solution at $37^{\circ} \mathrm{C}$ for $30 \mathrm{~min}$. Brain images were captured and the infarct size was measured.

\section{Detection using transmission electron microscopy (TEM)}

Five models in each group were randomly selected. After anesthesia, the brain tissue was quickly retrieved at a low temperature. The infarct tissue or corresponding area of the control group was washed, cut into $1 \times$ $1 \times 1 \mathrm{~mm}$ sections, and fixed with $3.5 \%$ glutaraldehyde at $4^{\circ} \mathrm{C}$. After rinsing with phosphate buffer, samples were placed in $1 \%$ tetraoxonium acid at $4^{\circ} \mathrm{C}$ for $24 \mathrm{~h}$, dehydrated using an alcohol-acetone stepwise gradient and subjected to penetration, embedding, semi-thin sectioning, and positioning using light microscopy. The area of interest was ultra-thinly sectioned followed by double staining with lead citrate-uranium acetate and observed using a transmission electron microscope (JEM-1011).

\section{RNA isolation and CDNA preparation}

Three models were randomly selected from each group. The infarct tissue was obtained as described above, placed in a pre-cooled $0.1 \%$ DEPC cryotube, and stored in a $-80^{\circ} \mathrm{C}$ refrigerator to prevent RNase contamination. RNA was isolated from the infarct tissue, the concentration was adjusted to $100 \mathrm{ng} / \mu \mathrm{L}$, and $20 \mu \mathrm{L}$ of complementary DNA was prepared in a reaction system. The mRNA was broken into short fragments and first-strand cDNA was synthesized using random primers. Buffer, dNTPs, and DNA polymerase I were added to the system to synthesize and purify the second-strand cDNA.

\section{RNA transcriptome sequencing analysis}

Illumina Hi-seq2500 platform was used for RNA-seq detection. All samples were tested for cross-species contamination. The read samples were compared to the tree shrew genome. The spliced unigene of the 
sample was compared with the publicly available gene expression data and functional annotation was performed through homology. Differential expression analysis between two conditions/groups (two biological replicates per condition) was performed using the DESeq R package (1.10.1). DESeq provides statistical routines for determining differential expression in digital gene expression data using a model based on a negative binomial distribution. The resulting P-values were adjusted using Benjamini and Hochberg's approach for controlling the false discovery rate. Genes with an adjusted P-value $<0.05$ found by DESeq were assigned as differentially expressed.

\section{Gene ontology (GO) and KEGG pathway enrichment analysis}

GO enrichment analysis of differentially expressed genes was performed using the GOseq R package, in which gene length bias was corrected. GO terms with a corrected P-value of less than 0.05 were considered significantly enriched by differentially expressed genes. KEGG is a database resource for understanding high-level functions and utilities of a biological system, such as a cell, organism, and ecosystem, from molecular-level information, especially large-scale molecular datasets generated using genome sequencing and other high-throughput experimental techniques (http://www.genome.jp/kegg/). We used KOBAS software to test the statistical enrichment of differentially expressed genes in KEGG pathways.

\section{Results}

\section{Rough comparisons}

In the IS and control groups, tree shrews consumed and excreted normal amounts of drinking water and urine, respectively, as well as showed normal activated movement and displayed shiny body hair. In the DM and DMIS groups, the model weights were significantly reduced compared to those in the control and IS groups. The animals in the DM and DMIS groups also had significantly increased water intake and urine volume compared to the control and IS groups. There was no significant difference between the control and IS groups.

\section{Detection of biochemical indicators}

The serum glucose levels of the DM and DMIS groups were significantly higher than those of the control and IS groups. Compared to the control group, the DM group had significantly higher levels of total protein, glucose, LDH1, CK-MB, and HBDH. Compared to the IS group, the DMIS group had higher GLOB and glucose levels after DM modeling (Table 1).

Table 1. Comparison of body weight and blood biochemical indexes among groups. (Mean \pm SD) 


\begin{tabular}{|c|c|c|c|c|c|c|}
\hline Indicators & $\begin{array}{l}\text { Control group } \\
n=18\end{array}$ & $\begin{array}{l}\text { DM group } \\
\mathrm{n}=18\end{array}$ & $\begin{array}{l}\mathrm{p} \\
\text { value }\end{array}$ & $\begin{array}{l}\text { IS group } \\
\mathrm{n}=18\end{array}$ & $\begin{array}{l}\text { DMIS group } \\
\mathrm{n}=18\end{array}$ & $\begin{array}{l}\mathrm{p} \\
\text { value }\end{array}$ \\
\hline $\begin{array}{l}\text { Initial } \\
\text { Body }\end{array}$ & $138.97 \pm 9.61$ & $131.44 \pm 11.82$ & 0.044 & $140.49 \pm 9.94$ & $134.32 \pm 15.73$ & 0.170 \\
\hline $\begin{array}{l}\text { weight* } \\
\text { Body } \\
\text { weight } \\
\text { after } \\
\text { stroke* }\end{array}$ & $141.15 \pm 11.76$ & $116.53 \pm 16.30$ & 0.000 & $137.99 \pm 14.36$ & $120.29 \pm 13.82$ & 0.001 \\
\hline $\begin{array}{l}\text { Total } \\
\text { protein }\end{array}$ & $52.96 \pm 3.62$ & $56.82 \pm 3.63$ & 0.003 & $52.38 \pm 3.55$ & $53.93 \pm 3.26$ & 0.181 \\
\hline ALB & $35.93 \pm 2.99$ & $36.31 \pm 5.86$ & 0.808 & $35.28 \pm 2.60$ & $35.66 \pm 4.22$ & 0.747 \\
\hline ALT & $207.88 \pm 289.94$ & $106.38 \pm 63.32$ & 0.163 & $150.88 \pm 115.06$ & $117.78 \pm 44.58$ & 0.267 \\
\hline GLOB* & $21.93 \pm 7.59$ & $22.23 \pm 7.40$ & 0.905 & $15.53 \pm 1.63$ & $23.21 \pm 7.34$ & 0.000 \\
\hline $\mathrm{Cr}$ & $14.00 \pm 6.75$ & $17.37 \pm 4.79$ & 0.094 & $14.63 \pm 4.12$ & $17.75 \pm 8.57$ & 0.176 \\
\hline UREA & $13.00 \pm 7.74$ & $11.55 \pm 3.07$ & 0.468 & $12.61 \pm 9.63$ & $11.96 \pm 3.99$ & 0.794 \\
\hline GLU & $5.38 \pm 0.52$ & $5.72 \pm 0.96$ & 0.198 & $5.17 \pm 0.41$ & $5.35 \pm 1.09$ & 0.519 \\
\hline $\begin{array}{l}\text { GLU after } \\
\text { DM } \\
\text { modeling* }\end{array}$ & $6.52 \pm 1.32$ & $26.75 \pm 10.60$ & 0.000 & $10.66 \pm 2.24$ & $32.29 \pm 6.08$ & 0.000 \\
\hline UA & $23.32 \pm 12.77$ & $19.86 \pm 12.97$ & 0.426 & $17.14 \pm 9.67$ & $13.71 \pm 11.60$ & 0.342 \\
\hline LDH1* & $6.97 \pm 3.99$ & $22.93 \pm 15.33$ & 0.000 & $41.70 \pm 62.45$ & $24.49 \pm 17.22$ & 0.273 \\
\hline CK-MB* & $42.50 \pm 15.78$ & $93.63 \pm 97.84$ & 0.042 & $88.50 \pm 60.11$ & $136.00 \pm 161.30$ & 0.254 \\
\hline $\begin{array}{l}\text { LDH } \\
\text { HBDH* }^{*}\end{array}$ & $\begin{array}{l}318.50 \pm 107.67 \\
356.50 \pm 90.40\end{array}$ & $\begin{array}{l}424.00 \pm 274.33 \\
470.25 \pm 136.92\end{array}$ & $\begin{array}{l}0.143 \\
\mathbf{0 . 0 0 6}\end{array}$ & $\begin{array}{l}418.25 \pm 113.26 \\
537.75 \pm 174.93\end{array}$ & $\begin{array}{l}437.50 \pm 206.40 \\
573.88 \pm 254.82\end{array}$ & $\begin{array}{l}0.731 \\
0.624\end{array}$ \\
\hline CK & $223.13 \pm 51.73$ & $222.88 \pm 95.36$ & 0.992 & $225.88 \pm 123.00$ & $264.38 \pm 79.54$ & 0.274 \\
\hline TB* & $19.60 \pm 8.15$ & $9.90 \pm 2.62$ & 0.000 & $12.51 \pm 3.85$ & $12.59 \pm 4.31$ & 0.954 \\
\hline AST & $252.09 \pm 130.15$ & $299.13 \pm 75.54$ & 0.196 & $232.50 \pm 119.14$ & $277.61 \pm 105.02$ & 0.237 \\
\hline CHOL* & $2.85 \pm 0.73$ & $7.40 \pm 3.26$ & 0.000 & $5.98 \pm 1.53$ & $7.80 \pm 3.41$ & 0.050 \\
\hline HDL-CH* & $1.84 \pm 0.40$ & $1.29 \pm 0.73$ & 0.009 & $1.59 \pm 0.53$ & $1.50 \pm 0.53$ & 0.614 \\
\hline LDL-CH* & $1.53 \pm 0.37$ & $2.93 \pm 0.70$ & 0.000 & $2.98 \pm 0.79$ & $3.06 \pm 0.95$ & 0.785 \\
\hline TG* & $1.02 \pm 0.45$ & $1.93 \pm 0.63$ & 0.000 & $1.63 \pm 0.47$ & $1.73 \pm 0.29$ & 0.449 \\
\hline CRP* & $0.51 \pm 0.24$ & $1.18 \pm 0.37$ & 0.000 & $1.19 \pm 0.47$ & $1.43 \pm 0.53$ & 0.160 \\
\hline TPT & $1.67 \pm 1.87$ & $0.72 \pm 0.61$ & 0.053 & $0.70 \pm 0.49$ & $0.77 \pm 0.52$ & 0.680 \\
\hline MYO* & $63.91 \pm 53.90$ & $36.36 \pm 12.00$ & 0.048 & $66.35 \pm 51.26$ & $45.86 \pm 21.80$ & 0.132 \\
\hline
\end{tabular}

Note: * and bold mean there is a statistical difference.

\section{TTC staining}

After TTC staining, there was no obvious abnormality in the brain tissue of the control or DM groups. In the IS group, obvious infarcts were observed in the lighted area of the cerebral cortex with clear boundaries. The infarct area accounted for $16.16 \pm 2.06 \%$ of the hemisphere. In the DMIS group, the infarct size was significantly larger than that in the IS group (19.56 $\pm 1.25 \%$; Table 2; Figure S1A).

Table 2. The cortical infarct size of tree shrew among groups (Mean \pm SD)

\begin{tabular}{lcc}
\hline Groups & Number & Infarct size (\%) \\
\hline Control & 5 & - \\
\hline DM & 5 & - \\
IS & 5 & $16.16 \pm 2.06$ \\
\hline DM+IS & 5 & $19.56 \pm 1.25^{*}$ \\
\hline
\end{tabular}


*P॰0.05 represents a significant difference between DM+IS group and IS group.

\section{$\mathrm{H}-\mathrm{E}$ staining}

After H-E staining, brain tissue in the control group showed an intact structure and the neural cells had normal morphology, abundant cytoplasm, and no obvious nuclear shrinkage. The brain tissue of the DM group was not obviously different from that of the control group. Nerve cells had normal morphology without edema and were in a tight arrangement. In the IS group, neural cells in the cerebral cortex showed a disorderly arrangement, and pyramidal cells showed a triangular morphology and eosinophil staining. The nucleus appeared shrunken and the nucleolus was absent. A gap had formed around the cells and the boundaries of the nucleus and cytoplasm were unclear. The findings in the DMIS group were similar to those in the IS group in terms of structure. Most cells lacked an entire cell structure (Figure S1B).

\section{TEM detection}

In the control group, the neural cells of the cortex were normal in terms of morphology and intact with clear nuclear membrane and nucleolus. The boundary of the neural cells was clear and the chromatin was abundant. The endoplasmic reticulum was arranged regularly and the ribosomes were also clear and intact. In the DM group, the perinuclear space of the neural cells was narrowed and the nucleoli were dense. The heterochromatin of cells was reduced and the mitochondria were swollen. The Golgi complex was expanded and the ribosomes of the rough endoplasmic reticulum were partially swollen and shed. Glial cells were observed around the thickening capillaries (Figure S2).

In the IS group, the neural cells in the cerebral ischemic area showed pyknosis and some cells showed edema. The chromatin of the cells condensed into clumps and the organelle structure was indistinct. Swollen mitochondria and expanded endoplasmic reticulum were also observed. In the DMIS group, a large number of neural cells displayed pyknosis with irregular nuclei and increased heterochromatin. The cytoplasm was concentrated with an indistinct organelle structure and a large amount of debris. Mitochondria were swollen and the crests dissolved and absent. The endoplasmic reticula were also swollen and expanded (Figure S2).

\section{RNA-seq analysis}

In this study, RNA sequencing was used to harvest transcriptomes of the control, IS, DM, and DMIS groups. A total of 12 sequencing libraries were prepared. The clean bases generated in each group were as follows: control group, 21.09G; IS group, 20.6G; DM group, 20.66G; and DMIS group, 21.81G. After removing the lowquality readings, a clean reading of greater than $95 \%$ was obtained on the Phred quality score Q20 level, and a clean reading greater than $90 \%$ was obtained on the Q30 level. The GC content was approximately $50 \%$ (Figure 3).

Analysis of differentially expressed genes between groups showed that there were 769 differentially expressed genes, including 508 upregulated and 261 downregulated genes between the DM and control groups. The top three upregulated and downregulated genes are listed in Table 3. There were 2206 
differentially expressed genes, including 1118 upregulated and 1088 downregulated genes, between the IS and DMIS groups. The top three upregulated and downregulated genes are listed in Table 4.

Table 3. DM group compared with control group (up and down) the difference in the number of the first 3 genes

\begin{tabular}{cccl}
\hline Gene id & log2FoldChange & padj & \multicolumn{1}{c}{ Blast swiss prot } \\
\hline 102471344 & 8.0682 & $1.36 \mathrm{E}-18$ & IL-8 \\
102473006 & 7.5287 & 0.00032122 & C-C motif chemokine 2 \\
102474225 & 7.2666 & $1.62 \mathrm{E}-100$ & Alpha-1-antichymotrypsin \\
Novel00254 & -3.5919 & 0.030012 & $-/ /-$ \\
102482464 & -3.2752 & $2.35 \mathrm{E}-09$ & BTB/POZ domain-containing protein 17 \\
Novel00985 & -3.2435 & 0.013019 & Ceruloplasmin \\
\hline
\end{tabular}

Table 4. DM + cerebral ischemia group and cerebral ischemia group (up and down) the difference in the number of the first three genes

\begin{tabular}{cccl}
\hline Gene id & log2FoldChange & padj & \multicolumn{1}{c}{ Blast swiss prot } \\
\hline 102468910 & 6.4108 & $3.12 \mathrm{E}-06$ & C-C motif chemokine 7 \\
102481193 & 5.8272 & 0.013623 & ATP-binding cassette sub-family A member 12 \\
102481906 & 5.7727 & 0.017142 & Adhesion G protein-coupled receptor E2 \\
102503204 & -5.909 & 0.00209 & HLA class II histocompatibility antigen \\
Novel00985 & -3.7814 & $3.38 \mathrm{E}-06$ & Ceruloplasmin [Tupaia chinensis] \\
102492418 & -3.4827 & 0.030513 & Homeobox protein Hox-D1 \\
\hline
\end{tabular}

\section{Gene ontology and KEGG pathway enrichment analysis}

For GO enrichment analysis, there was no significant difference in the biological process (BP), cellular component (CC), and molecular function (MF) of downregulated genes between the DM and control groups (padj > 0.05). Among the upregulated genes, BP annotation was mainly in the immune response (padj < 0.05), and the genes involved included C-C motif chemokine 24 (CCL24), C-X-C motif chemokine ligand 2 (CXCL2, also known as stroma-derived factor 1), oncostatin-M, CCL15, and protein FAM83G. For the immune system process (padj $<0.05$ ), the genes included tetratricopeptide repeat protein $7 \mathrm{~A}$, tubulointerstitial nephritis antigen, interleukin-8 (IL-8), and IL-6. The CC annotations were mainly in the extracellular region (padj < 0.05). The genes involved included fibronectin, oncostatin-M, and CCL15. For the extracellular matrix (padj < 0.05), the genes included S100-A12 protein, collagen alpha-3(V) chain, and disintegrin and metalloproteinase domain-containing protein 8 . The MF annotations were mainly in cytokine activity (padj < 0.05), which included CXCL2, leukemia inhibitory factor, platelet basic protein, and CCL7. Chemokine activity (padj < 0.05) included CCL2, CCL7, platelet basic protein, and CXCL2. Chemokine receptor binding (padj < 0.05 ) included CCL24, IL-8, CXCL2, and CCL15. G protein-coupled receptor binding (padj < 0.05 ) included CXCL2, CCL15, CCL2, CCL24, and IL-8. Receptor binding (padj < 0.05) included Wnt-5b, SPRY domaincontaining SOCS box protein 1, and Wnt-9a. Cytokine receptor binding (padj < 0.05 ) included CCL2, serine/threonine-protein kinase RIO3, CCL15, and CXCL2. Protein binding (padj < 0.05) included enhancer of filamentation 1, neutrophil cytosol factor 4, and telomeric repeat-binding factor 1 (Figures 4A and 4B).

There was also no significant difference in the BP, CC, and MF of downregulated genes between the IS and DMIS groups (padj > 0.05). The upregulated BP was mainly involved in inflammatory response molecular 
mediator production (padj < 0.05), and the genes involved included IL-21 receptor, IL-31-a-receptor subunit, and IL-6 receptor subunit beta. The upregulated MF annotation was mainly in chemokine activity (padj < 0.05), including CCL15, platelet basic protein, CCL2, and CCL7. Chemokine receptor binding (padj < 0.05 ) included CCL24, CXCL2, and CXCL2. Cytokine activity (padj < 0.05 ) included platelet basic protein, CCL15, CCL2; and cytokine receptor activity (padj $<0.05$ ). The main genes involved were IL-31 receptor subunit, IL-21 receptor, and IL-6 receptor subunit $\beta$ (Figures 4C and 4D).

KEGG analysis found that there were 14 differential signaling pathways for upregulated genes between the $\mathrm{DM}$ and control groups, including cytokine-cytokine receptor interaction, ECM-receptor interaction, NF-KB signaling pathway, MAPK signaling pathway, TNF signaling pathway, and PI3K-Akt signaling pathway. There were no differential signaling pathways for downregulating genes (Figures $5 \mathrm{~A}$ and $5 \mathrm{~B}$ ).

There was one differential signaling pathway based on upregulated genes (padj < 0.05 ) between the DMIS and IS groups, which were proteoglycans in cancer. There were seven differential signaling pathways based on downregulated genes (padj < 0.05), including the glutamatergic synapse, axon guidance, and gastric acid secretion (Figures 5C and 5D).

\section{Discussion}

At present, the prevention and treatment of stroke in patients with DM are similar to those in patients without DM. However, patients with DM have a higher risk of stroke and poorer neurological recovery prognosis than patients without DM. It is, thus, necessary to develop specific stroke prevention and treatment strategies for patients with DM. Although the mechanism is not yet completely understood, the hyperglycemia status may affect the integrity of white matter and the generation of oligodendrocytes after a stroke, as well as affect inflammation in the neural microenvironment and vascular permeability (Zuloaga et al, 2014; MitomeMishima et al, 2014; (Venkat et al, 2018). In this study, we innovatively implemented changes in the transcriptome of neural tissue in DM and control tree shrew models and analyzed the changes after stroke in the IS and DMIS models using RNA-seq analysis. This study aimed to provide a theoretical basis for the prevention and treatment of stroke in patients with DM.

Due to the depletion of non-human primate resources, this study adopted tree shrews with biological characteristics similar to those of rhesus monkeys. Tree shrews are the closest animals to humans in terms of anatomical and physiological characteristics compared to other rodents (Kaas et al, 2013). The structure of the brain and distribution of intracranial cerebral blood vessels of tree shrews are close to those of humans and most of the intracortical arteries are linearly distributed with a dense capillary network (Zhang at el, 2017). Therefore, the tree shrew IS model had pathological processes similar to those of clinical stroke patients. In the DM model, blood glucose increased significantly and affected body weight, water intake, and urine volume. After IS onset, the infarct size in the brains of the DMIS group significantly increased compared to that in the IS group. TEM results showed that DM increased the loss in the number of neural cells and the degree of damage.

Under acute stress, the stress-induced hyperglycemia is a common physiological response that usually refers to transient hyperglycemia (Yuan et al. 2021). In the population, stress-induced hyperglycemia occurs 
in approximately half of patients with acute ischemic stroke, and it is generally believed to be associated with an increased risk of death and poor neurological prognosis (Li et al. 2020). The potential mechanism included hypothalamic-pituitary-adrenal (HPA) axis activation, glucocorticoids release and the sympathetic nervous system activation, that leading to catecholamine-mediated hyperglycemia. But hyperglycemia has a dual role in brain injury. On the one hand, high glucose provides more energy supply for the brain, but it also exacerbates neuronal damage (Zhang et al. 2018). In this study, the blood glucose of IS group was significantly higher than that of the control group. In further research, it is worth to conduct a study of RNAseq analysis on whether there is a hyperglycemia after the ischemia stroke to provide clues for intervention of stress-induced hyperglycemia.

In addition to stress-induced hyperglycemia, clinical studies have found that hyperglycemia/ diabetes are also associated with poor prognosis after ischemia or hemorrhagic stroke that including higher mortality, poor neurological functional prognosis, longer hospital stay, readmission and recurrence rate (Lau et al. 2019). In a meta-analysis, diabetes was considered as an independent predictor of recurrence in stroke patients that recurrence rate of stroke patients with diabetes are significantly higher than that of patients without diabetes (HR: 1.45; 95\% Cl: 1.32-1.59) (Shou et al. 2015). And stroke patients with diabetes have more serious neural tissue damage and larger infarct size, and in-vitro study have confirmed that high glucose levels increase neural cells apoptosis (Bai et al. 2021). It is consistent with the results of our research that compared to IS group, DMIS group has larger infarct area, more neural cells loss, and more server neural tissue damage.

RNA-seq showed that by comparing the DM and control group, the expression of IL-8, CC motif chemokine 2, and alpha-1-antichymotrypsin was increased in the DM group. Two of these three genes were involved in the inflammatory response (Figure $6 \mathrm{~A}$ ). BP of GO annotation also found that upregulated genes are involved in the immune response and immune system processes, and MF found that genes are mostly involved in cytokine and chemokine activities. IL-8 is a chemokine that has a chemotactic effect on inflammatory cells, such as neutrophils, basophils, and T cells, and is also involved in the activation of neutrophils. The increased expression of IL-8 in the DM model causes inflammatory cells, such as neutrophils, to accumulate in the nervous system, and induces a pre-inflammatory state in the nervous tissue. IL-8 has a potential correlation with the incidence of first stroke (Shishkova et al, 2018囚Jenny et al, 2019). This may be one of the mechanisms by which patients with DM have a high risk of IS.

A recent study also found that the number of granulocytes and natural killer cells is related to brain damage after a stroke and that IL-8 could attract these two types of cells. Peripheral IL-8 levels are also considered to be related to infarct size and prognosis (He et al, 2018). In a mouse IS model, the expression of IL-8 in peripheral mononuclear cells could affect the occurrence of IS by regulating the PI3K/Akt/NF-kb signaling pathway. After reducing the expression of IL-8, it reduced the infarct size, activated the neurogliocytes, and improved neural function recovery (Lv et al, 2019). This study further suggests that IL-8 expression in the brain increases in patients with DM, as do the activities of the NF-KB, MAPK, TNF, and PI3K-Akt signaling pathways. Therefore, in patients with DM, the increased expression of IL-8 and the activities of the inflammatory status in the neural system may also be a cause of poor neurological function prognosis after IS onset. Therefore, for the prevention and treatment of stroke in patients with DM, not only blood glucose 
concentration but also the inflammatory status of the central nervous system must be reduced. This may allow the reduction of the incidence of stroke and improvement of the prognosis of neurological function in patients with DM.

In comparison with the IS and DMIS models, the expression of CCL7, ATP-binding cassette sub-family A member 12, and adhesion G protein-coupled receptor E2 increased (Figure 6B). Adhesion G protein-coupled receptor E2 promotes chemotaxis and adhesion of granulocytes, promoting the release of inflammatory factors, such as IL-8 and TNFs, and increases the activity of the intracranial inflammatory response. CCL7 can also attract monocytes and eosinophils. In a mouse model, CCL7 expressed in meningeal mast cells could participate in the pathological process of stroke, including the infiltration of granulocytes and activation of macrophages, and further lead to an increase in brain swelling and infarct size (Arac et al, 2014). The CCL7 and adhesion G protein-coupled receptor E2-related high inflammatory response may also be a cause of worse neurological outcomes in patients with DM after IS.

Owing to the use of DM and IS models in tree shrews, there were differences in the actual pathogenesis of patients. However, RNA-seq studies on clinical patients with DM and IS are not yet available. Therefore, this study could be used as a reference for further clinical and basic research. This study was focus on the GO and KEGG pathway enrichment analysis based on RNA-seq analysis in design, but not the single gene expression level. So, we ignored the design of RT-PCR verification of differentially expressed genes. This was a limitation of this work.

In conclusion, this study confirmed that in a tree shrew DM model, increased expression of IL-8 and activation of the inflammatory status may be causes of increased IS susceptibility, and high CCL7-related inflammatory response may also be a cause of poor neurological prognosis after IS. Therefore, in the prevention and treatment of stroke in patients with $\mathrm{DM}$, not only reducing blood glucose levels but also reducing the inflammatory state of the nervous system may be a method of reducing the incidence of stroke and improving the prognosis of neurological function after IS.

\section{Declarations}

Funding: This work was supported by grants from National Science Fund subsidized project (81760327), Yunnan Province Young and Middle-aged Academic and Technical Leaders Reserve Talents Training Project(2017HB049), Yunnan high-level health and family planning technical personnel training project $(\mathrm{H}-$ 2017043), Yunnan Provincial Department of Science and Technology - Kunming Medical Joint Project (2018FE001(-228)), Kunming Medical University Second Affiliated Hospital Talent Training Program (RCPYXM2017-3-16), Research Institutions Program in Yunnan medical and health units(2018NS0293). Kunming Medical University Education Research and Reform Program (2020-JY-Y-085). The funders had no role in study design, data collection and analysis, decision to publish, or preparation of the manuscript.

Conflicts of interest: The authors have no relevant financial or non-financial interests to disclose.

Availability of data and material (data transparency): The datasets generated during and/or analysed during the current study are available from the corresponding author on reasonable request. 


\section{Code availability: Not applicable}

\section{Author contribution:}

Ling Zhao and Tingyu Ke: study concept and design, analysis and interpretation of data, drafting of the manuscript, funding acquisition. Ling Zhao, Shiying Huang, and Qiwei Liao: the acquisition of samples or data, statistical analysis, review of the manuscript. Ling Zhao, Xia Li, Shufen Tan, and Shuqing Li: statistical analysis, review of the manuscript, material supports

\section{Acknowledgement:}

Thank Professor Jiejie Dai's laboratory. Thank Yuanyuan Han for providing suggestions and instructions in the process of modelling. Thank Ying Tang for helping us review pathology materials.

\section{References}

Arac A, Grimbaldeston MA, Nepomuceno AR, Olayiwola O, Pereira MP, Nishiyama Y, et al. Evidence that meningeal mast cells can worsen stroke pathology in mice. Am J Pathol. 2014;184(9):2493-504.

Bai B, Li D, Xue G, Feng P, Wang M, Han Y, et al. The novel GLP-1/GIP dual agonist DA3-CH is more effective than liraglutide in reducing endoplasmic reticulum stress in diabetic rats with cerebral ischemia-reperfusion injury. Nutr Metab Cardiovasc Dis. 2021;31(1):333-43.

Beckman JA, Paneni F, Cosentino F, Creager MA. Diabetes and vascular disease: pathophysiology, clinical consequences, and medical therapy: part II. Eur Heart J. 2013;34(31):2444-52.

Bhalla A, Wang Y, Rudd A, Wolfe CD. Differences in outcome and predictors between ischemic and intracerebral hemorrhage: the South London Stroke Register. Stroke. 2013;44(8):2174-81.

Bruno A, Liebeskind D, Hao Q, Raychev R, Investigators US. Diabetes mellitus, acute hyperglycemia, and ischemic stroke. Curr Treat Options Neurol. 2010;12(6):492-503.

Collaborators GBDS. Global, regional, and national burden of stroke, 1990-2016: a systematic analysis for the Global Burden of Disease Study 2016. Lancet Neurol. 2019;18(5):439-58.

He Q, Shi X, Zhou B, Teng J, Zhang C, Liu S, et al. Interleukin 8 (CXCL8)-CXC chemokine receptor 2 (CXCR2) axis contributes to MiR-4437-associated recruitment of granulocytes and natural killer cells in ischemic stroke. Mol Immunol. 2018;101:440-9.

Hill MD. Stroke and diabetes mellitus. Handb Clin Neurol. 2014;126:167-74.

Hong P, Gu RN, Li FX, Xiong XX, Liang WB, You ZJ, et al. NLRP3 inflammasome as a potential treatment in ischemic stroke concomitant with diabetes. J Neuroinflammation. 2019;16(1):121.

Jenny NS, Callas PW, Judd SE, McClure LA, Kissela B, Zakai NA, et al. Inflammatory cytokines and ischemic stroke risk: The REGARDS cohort. Neurology. 2019;92(20):e2375-e84. 
Jiang Y, Liu N, Wang Q, Yu Z, Lin L, Yuan J, et al. Endocrine Regulator rFGF21 (Recombinant Human Fibroblast Growth Factor 21) Improves Neurological Outcomes Following Focal Ischemic Stroke of Type 2 Diabetes Mellitus Male Mice. Stroke. 2018;49(12):3039-49.

Kaas JH, Gharbawie OA, Stepniewska I. Cortical networks for ethologically relevant behaviors in primates. Am J Primatol. 2013;75(5):407-14.

Kruyt ND, Biessels GJ, Devries JH, Roos YB. Hyperglycemia in acute ischemic stroke: pathophysiology and clinical management. Nat Rev Neurol. 2010;6(3):145-55.

Lau LH, Lew J, Borschmann K, Thijs V, Ekinci El. Prevalence of diabetes and its effects on stroke outcomes: A meta-analysis and literature review. J Diabetes Investig. 2019;10(3):780-92.

Li J, Quan K, Wang Y, Zhao X, Li Z, Pan Y, et al. Effect of Stress Hyperglycemia on Neurological Deficit and Mortality in the Acute Ischemic Stroke People With and Without Diabetes. Front Neurol. 2020;11(1):576895.

Liu P, Liu S, Feng N, Wang Y, Gao Y, Wu J. Association between neurological deterioration and outcomes in patients with stroke. Ann Transl Med. 2020;8(1):4.

Lv H, Li J, Che YQ. CXCL8 gene silencing promotes neuroglial cells activation while inhibiting neuroinflammation through the PI3K/Akt/NF-kappaB-signaling pathway in mice with ischemic stroke. J Cell Physiol. 2019;234(5):7341-55.

Ma S, Wang J, Wang Y, Dai X, Xu F, Gao X, et al. Diabetes Mellitus Impairs White Matter Repair and LongTerm Functional Deficits After Cerebral Ischemia. Stroke. 2018;49(10):2453-63.

Mitome-Mishima Y, Miyamoto N, Tanaka R, Shimosawa T, Oishi H, Arai H, et al. Adrenomedullin deficiency and aging exacerbate ischemic white matter injury after prolonged cerebral hypoperfusion in mice. Biomed Res Int. 2014;2014:861632.

Pintana H, Lietzau G, Augestad IL, Chiazza F, Nystrom T, Patrone C, et al. Obesity-induced type 2 diabetes impairs neurological recovery after stroke in correlation with decreased neurogenesis and persistent atrophy of parvalbumin-positive interneurons. Clin Sci (Lond). 2019;133(13):1367-86.

Romero JR, Morris J, Pikula A. Stroke prevention: modifying risk factors. Ther Adv Cardiovasc Dis. 2008;2(4):287-303.

Shishkova VN, Adasheva TV, Remenik AY, Valyaeva VN, Shklovsky VM. [Prognostic significance of clinicalanthropometric, biochemical, metabolic, vascular-inflammatory and molecular-genetic markers in the development of the first ischemic stroke]. Zh Nevrol Psikhiatr Im S S Korsakova. 2018;118(2):4-11.

Shou J, Zhou L, Zhu S, Zhang X. Diabetes is an Independent Risk Factor for Stroke Recurrence in Stroke Patients: A Meta-analysis. J Stroke Cerebrovasc Dis. 2015;24(9):1961-8. 
Venkat P, Yan T, Chopp M, Zacharek A, Ning R, Van Slyke P, et al. Angiopoietin-1 Mimetic Peptide Promotes Neuroprotection after Stroke in Type 1 Diabetic Rats. Cell Transplant. 2018;27(12):1744-52.

Wen L, Zhang S, Wan K, Zhang H, Zhang X. Risk factors of haemorrhagic transformation for acute ischaemic stroke in Chinese patients receiving intravenous thrombolysis: A meta-analysis. Medicine (Baltimore). 2020;99(7):e18995.

Yuan C, Chen S, Ruan Y, Liu Y, Cheng H, Zeng Y, et al. The Stress Hyperglycemia Ratio is Associated with Hemorrhagic Transformation in Patients with Acute Ischemic Stroke. Clin Interv Aging. 2021;16(1):431-42.

Zhang CR, Yu ZC, Li SQ. Mechanism of ischemic postconditioning relieved brain edema and cerebral infarction after cerebral ischemia in tree shrews. Acta Anatomica Sinica. 2017:48(2):135-41.

Zhang S, An Q, Wang T, Gao S, Zhou G. Autophagy- and MMP-2/9-mediated Reduction and Redistribution of ZO-1 Contribute to Hyperglycemia-increased Blood-Brain Barrier Permeability During Early Reperfusion in Stroke. Neuroscience. 2018;377(1):126-37.

Zhao L, Tan SF, Ke TY, Li SQ. Regulation of ischemic postconditioning on metabolic disorders of type 2 diabetes mellitus combined with cerebral ischemia in tree shrews. China Medical Abstracts(Internal Medicine). 2019:35(1):58-66.

Zuloaga KL, Krasnow SM, Zhu X, Zhang W, Jouihan SA, Shangraw RE, et al. Mechanism of protection by soluble epoxide hydrolase inhibition in type 2 diabetic stroke. PLoS One. 2014;9(5):e97529.

\section{Figures}




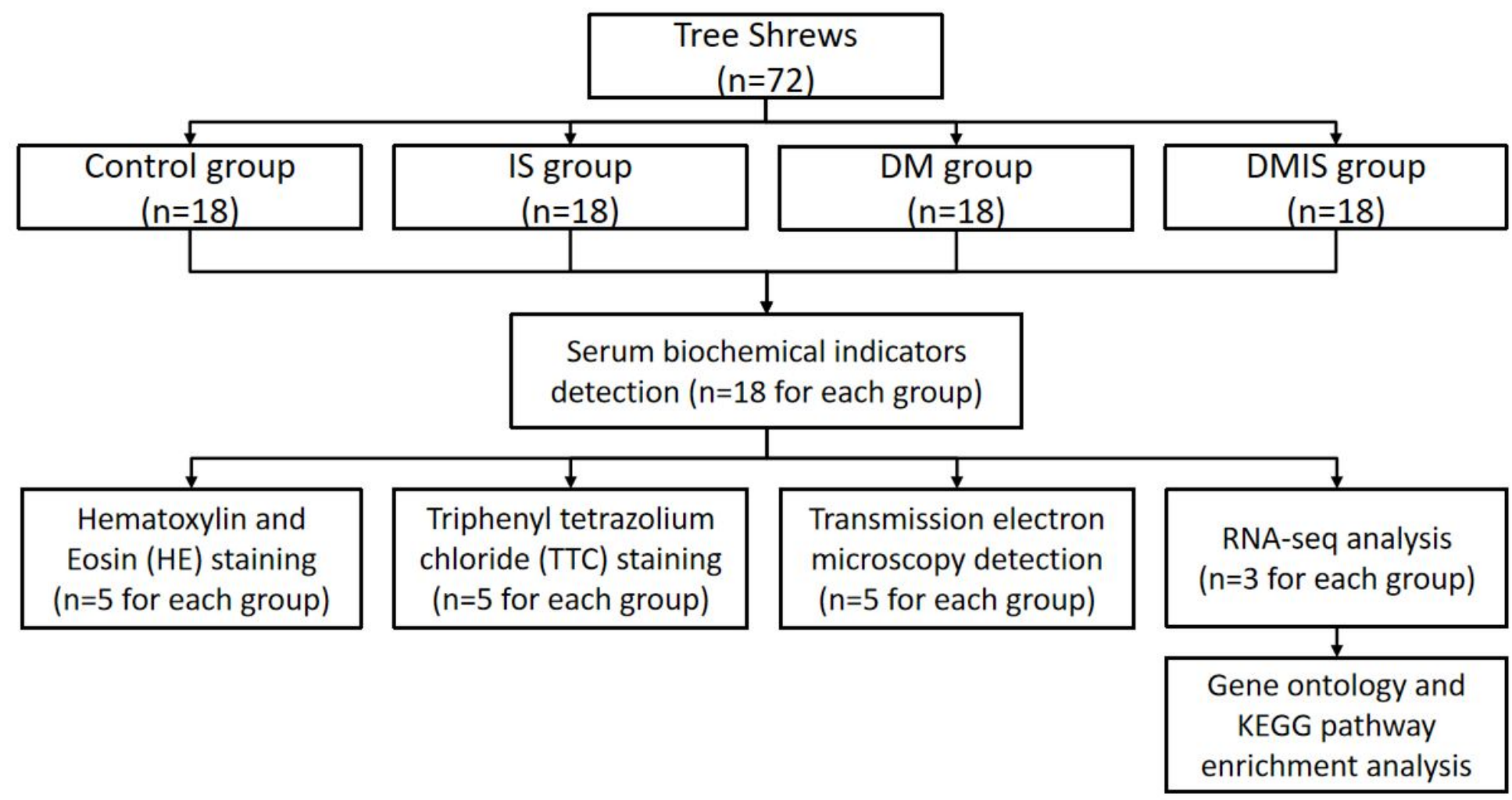

Figure 1

The outline of animal grouping and experimental designs
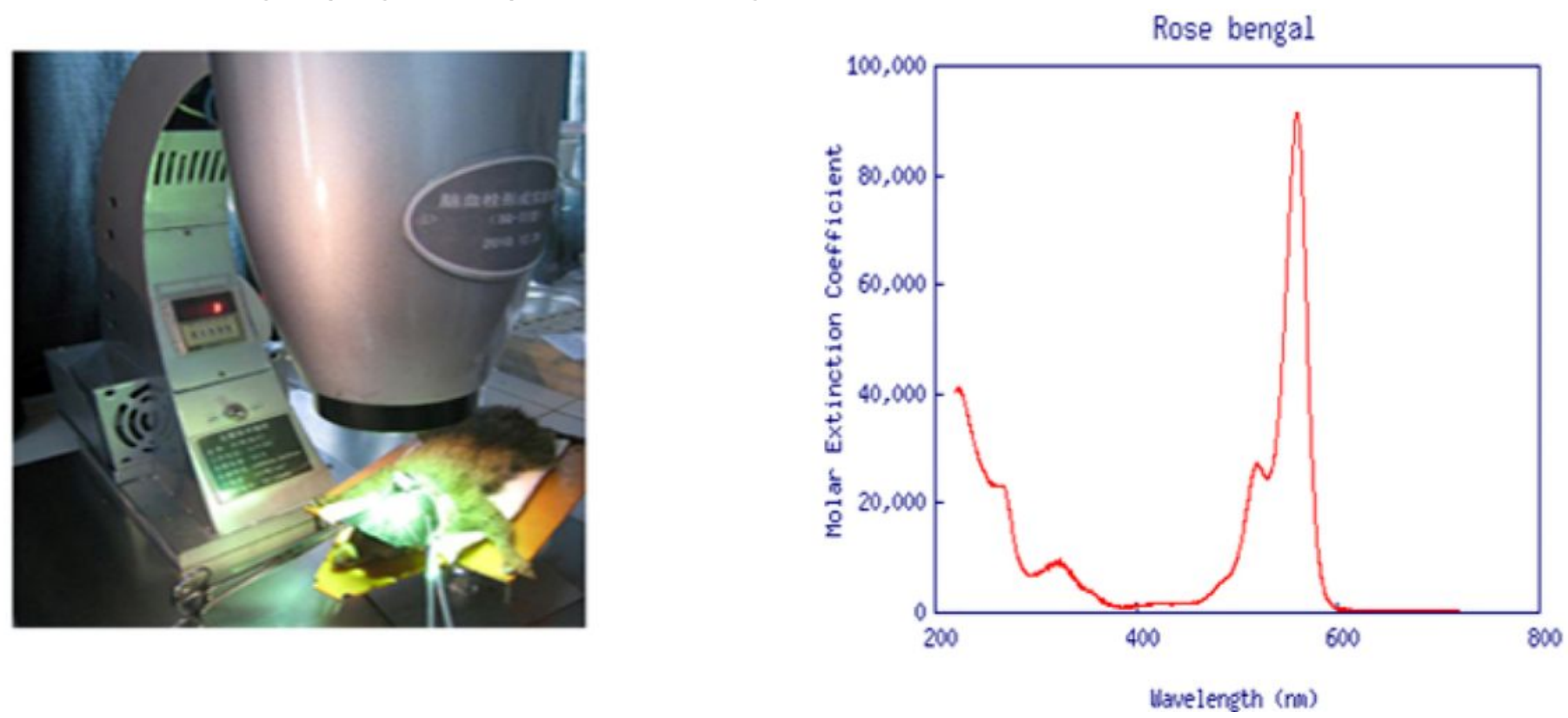
Figure 2

The establishment of the cortical thrombotic cerebral ischemia model in tree shrews with an SQ- $\mathbb{\text { cerebral }}$ thrombosis device.
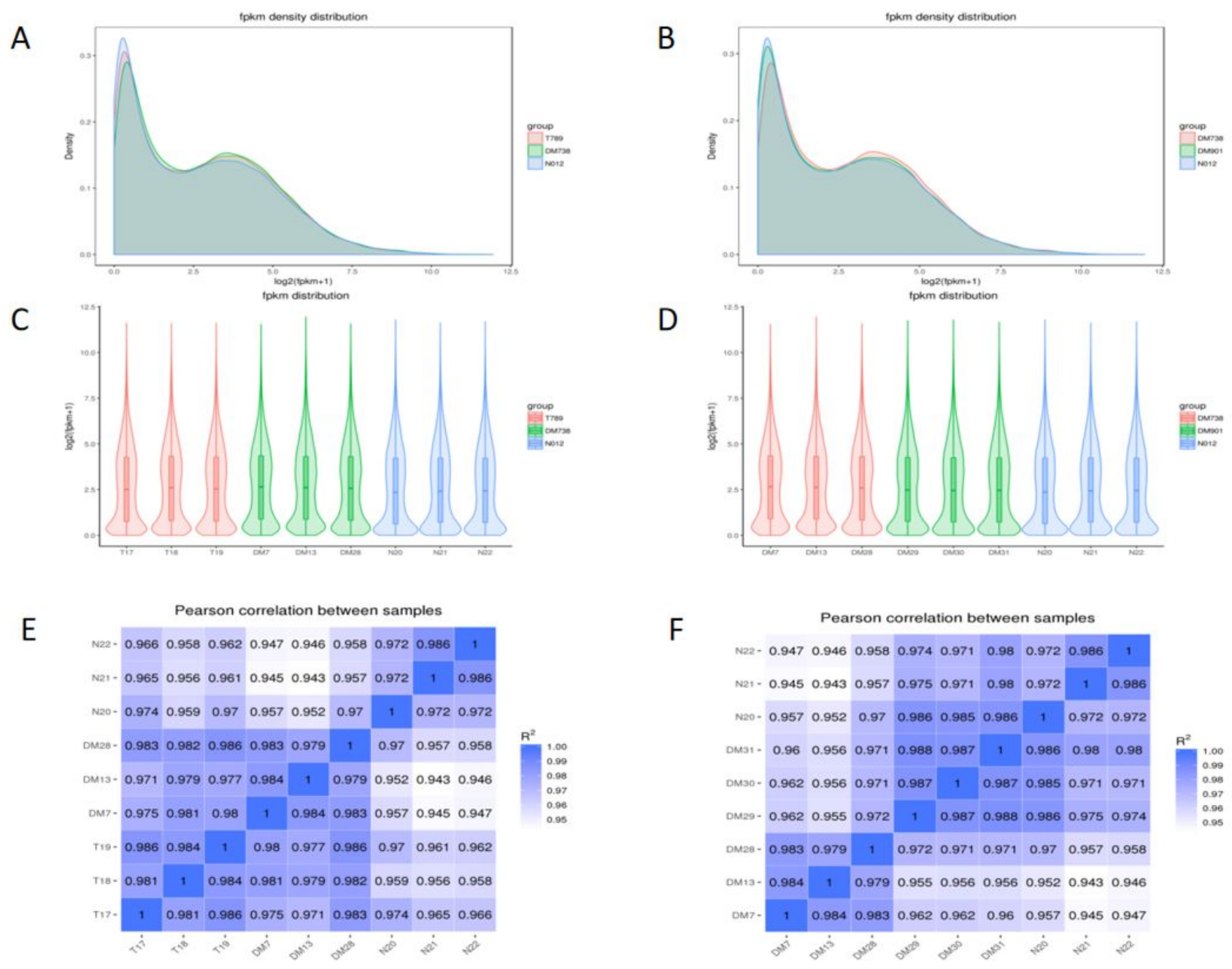

\section{Figure 3}

Fragments per kilobase million density distribution plot (A, B), violin plot (C, D) of each group, and Pearson correlation coefficient of RNA-seq results among groups (E, F). The control group (N012) consists of N20, N21, and N22; the IS group (T789) consists of T17, T18, and T19; the DM group (D901) consists of DM29, DM30, and DM31; and the DMIS group (D738) consists of DM7, DM13, and DM28. 

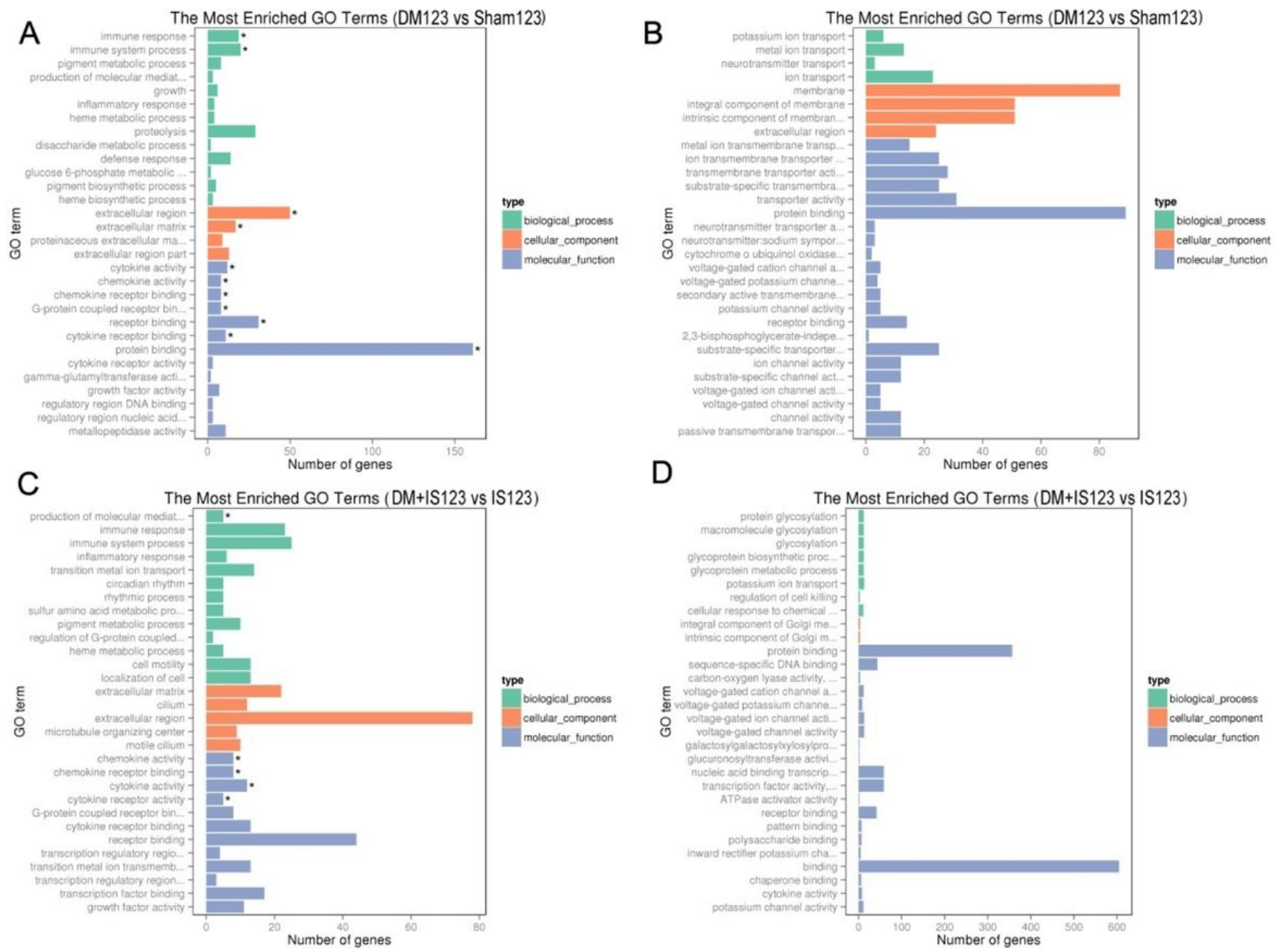

\section{Figure 4}

GO analysis results of differential genes between the DM and control groups (A: based on upregulated genes; B: based on downregulated genes); GO analysis results of differential genes between the IS and DMIS groups (C: based on upregulated genes; D: based on downregulated genes). 

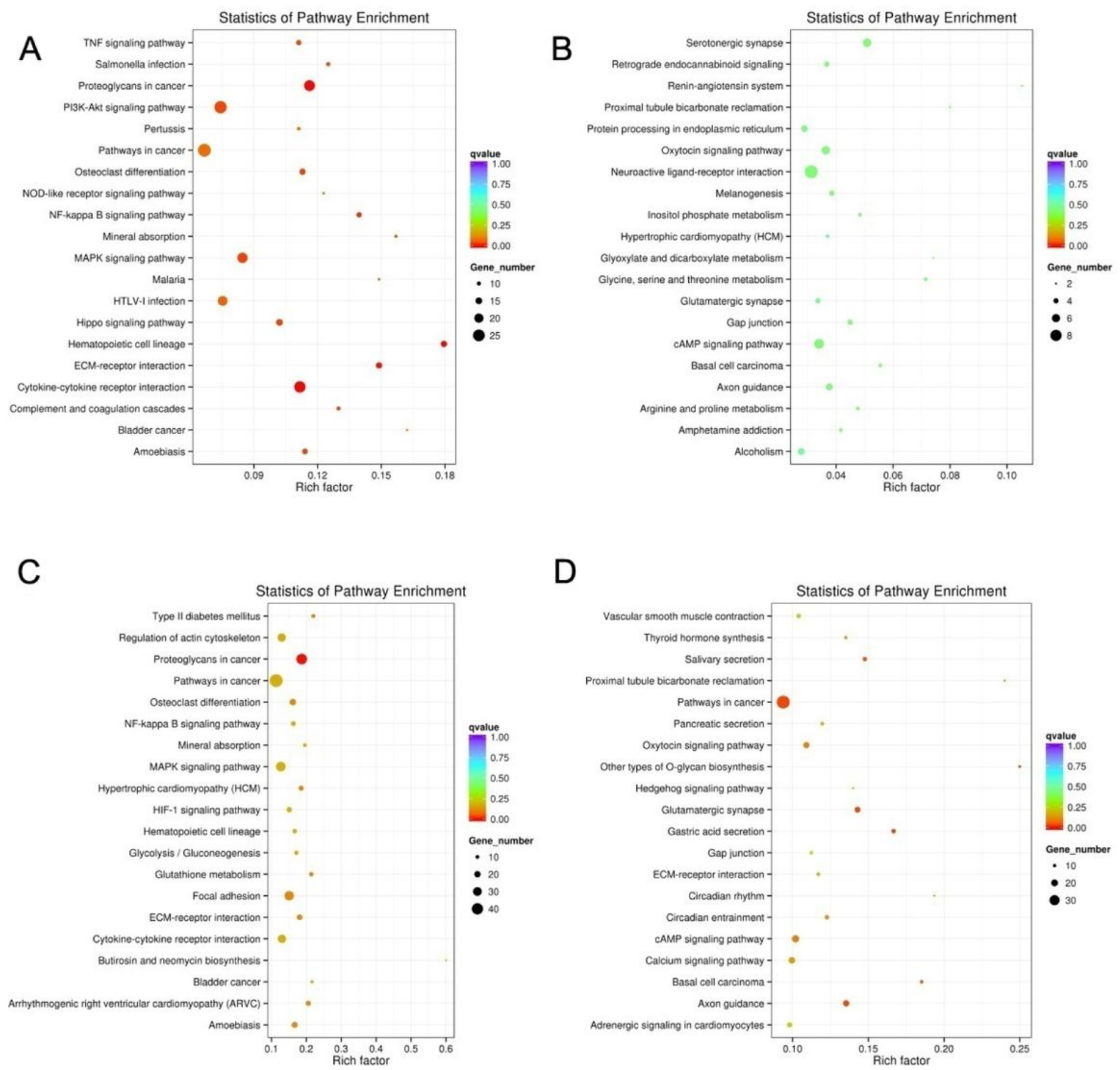

\section{Figure 5}

The differential signal pathways between the DM and control groups using KEGG analysis. (A: based on upregulated genes; $\mathrm{B}$ : based on downregulated genes). The differential signal pathways between the IS and DMIS groups using KEGG analysis. (C: based on upregulated genes; D: based on downregulated genes). The size of the spot reflects the number of genes involved and the color reflects the degree of statistical difference. 
A

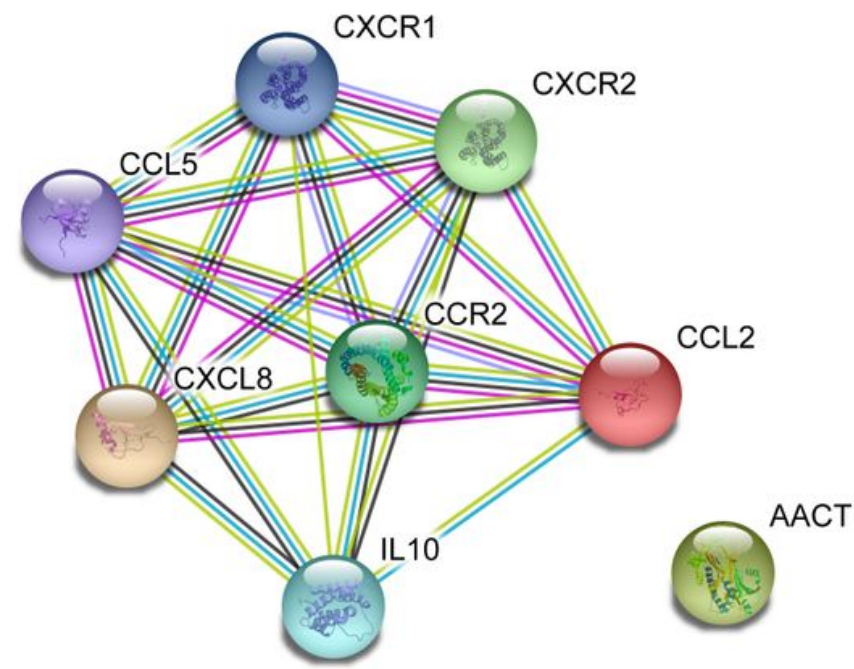

B

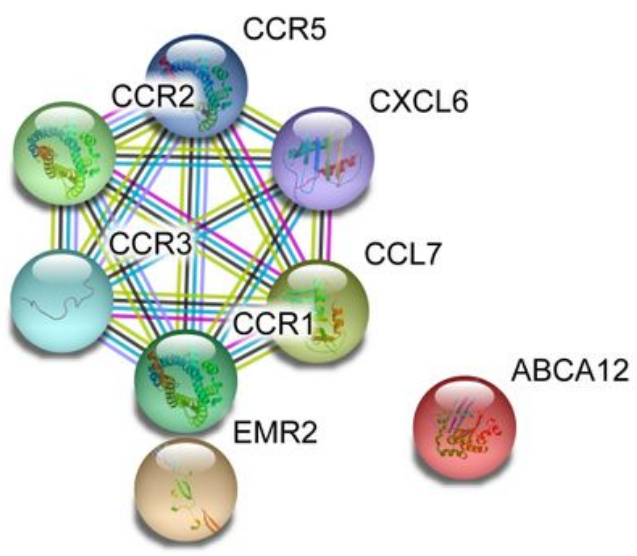

Figure 6

Protein-protein interaction plots of upregulated expressed genes and their interacting genes, A: between the DM and control groups; B: between the DMIS and IS groups.

\section{Supplementary Files}

This is a list of supplementary files associated with this preprint. Click to download.

- FigureS11TTCHEmodified.tif

- FigureS2TEM.tif 